\title{
Galectin-3 may serve as a potential marker for diagnosis and prognosis in papillary thyroid carcinoma: a meta-analysis
}

This article was published in the following Dove Press journal:

OncoTargets and Therapy

22 January 2016

Number of times this article has been viewed

\author{
Weiwei Tang' \\ Congwei Huang ${ }^{2}$ \\ Chongyin Tang' \\ Jin $\mathrm{Xu}^{\prime}$ \\ Hanjin Wang' \\ 'Department of General Surgery, \\ ${ }^{2}$ Department of Endocrinology, \\ Nanjing First Hospital, Nanjing \\ Medical University, Nanjing, Jiangsu, \\ People's Republic of China
}

Correspondence: Hanjin Wang

Department of General Surgery, Nanjing First Hospital, Nanjing Medical University, 68 Changle Road, Nanjing, Jiangsu 210006, People's Republic of China Tel +862552887042

Emailwhj_888@sohu.com
Background: Galectin-3 is a member of the beta-galactoside-binding protein family and functions as a modulator of cell growth through galactoside-binding protein correlated with the occurrence and metastasis of papillary thyroid carcinoma (PTC).

Methods: A systematic review of published articles on Web of Science and PubMed was performed. After establishing inclusion and exclusion criteria, nine articles were selected. Three studies referred to galectin-3 expression in PTC and non-PTC patients. Three studies referred to galectin-3 expression in PTC patients with lymph node metastasis (LNM) and without LNM. Three studies referred to galectin-3 expression in both PTC (with and without LNM) and nonPTC patients. Data analysis was performed by using RevMan5.2 software.

Results: A total of 424 patients from six eligible studies that provided data about galectin-3 expression in PTC and non-PTC patients were included. A total of 378 patients from six eligible studies that provided data about galectin-3 expression in PTC with LNM and without LNM were included. Immunohistochemistry technique was used in all the studies. Galectin-3 was found to be a highly sensitive $(275 / 424,64.86 \%)$ marker in the diagnosis of PTC, but was found to be expressed only in a few cases involving other types of thyroid lesions (58/424, 13.68\%). The odds ratio, expressed as PTC group versus other thyroid lesions group, was 13.97 (95\% CI: 7.51-26.01, $P<0.00001)$. The results also showed that the positive expression rates of galectin-3 in PTC patients with LNM were higher than those in PTC patients without LNM.

Conclusion: This meta-analysis demonstrated that galectin-3 may become a potentially useful immunomarker to distinguish between PTC and non-PTC patients. In addition, PTC patients with positive expression of galectin-3 were more prone to LNM.

Keywords: immunomarker, metastasis, thyroid lesions, immunohistochemistry

\section{Introduction}

Thyroid cancer is a common endocrine malignant tumor. Papillary thyroid carcinoma (PTC) is the most common histological subtype of thyroid cancers, accounting for approximately $75 \%-85 \%$ of all thyroid malignancies. ${ }^{1}$ Early diagnosis and prompt treatment of thyroid cancer may maximize the survival rate and prolong the survival time of patients. In recent years, advancements in molecular biology techniques have helped in providing early diagnosis as well as appropriate treatment for PTC. Researchers are now increasingly concerned about the biomarkers of PTC, including immunohistochemical markers. Galectin-3 is a member of galectin family that has an affinity for $\beta$-galactoside. ${ }^{2}$ It has been proposed to modulate cell adhesion and cell growth through its influence on the cell cycle., Due to its physiological roles, galectin-3 may act as an adhesion molecule in tumor progression and loosen 
the connection between tumor cells to promote cancer cell metastasis. ${ }^{5,6}$ Some studies have revealed that galectin-3 level is significantly elevated in cancer tissues and is associated with cancer metastasis. ${ }^{7-9}$

In the present study, all relevant articles about galectin-3 and PTC in the recent 5 years were collected. The aim of this study was to compare the positive expression rates of galectin-3 in patients with PTC and those in patients with other types of thyroid lesions. In addition, this research was also aimed to explore the relationship between galectin-3 expression levels and lymph node metastasis (LNM) and, furthermore, to determine whether galectin-3 can be used as a potential prognostic marker for the diagnosis of PTC.

\section{Methods}

\section{Identification}

This meta-analysis was performed in accordance with PRISMA (Preferred Reporting Items for Systematic Reviews and Meta-analyses). PubMed and Web of Science were searched for studies using both medical-subject-heading terms and free-text words. The following search terms were used: galectin-3, thyroid cancer, PTC, and metastasis. The last search was performed on July 28, 2015. The citation lists of the retrieved articles were manually screened to ensure the sensitivity of the search strategy.

\section{Inclusion and exclusion criteria}

Studies in this meta-analysis had to meet the following inclusion criteria: 1) inclusion of patients with PTC; 2) evaluation of the positive expression rates of galectin-3 in thyroid carcinoma; 3) publication as a full paper in English; and 4) inclusion of a control group. Studies were excluded based on the following criteria: 1) duplicate studies, reviews, letters, unpublished data, and comments; 2) published in a language other than English; 3) data that could not be extracted or calculated from the original article; and 4) nonhuman subjects.

\section{Data extraction}

The data of eligible studies were extracted in duplicate by two investigators independently (WWT and CWH). The following details were extracted: name of the first author, year of publication, country of study population, total number of patients included in the study, detection method, and positive or negative expression of galectin-3 in PTC with or without LNM. Two authors checked the extracted data and reached a consensus in consideration with all the collected data. Disputes were resolved by discussion. If dissent remained, then third and fourth investigators (CYT and JX) were consulted.

\section{Statistical analyses}

This meta-analysis was performed by using Review Manager (RevMan) Version 5.2 software (The Nordic Cochrane Centre, The Cochrane Collaboration, Copenhagen, Denmark). In all the studies, \pm and - were used to indicate negative expression, while,,++++++ , and ++++ were used for positive expression. Statistical heterogeneity among the studies was assessed by using the $I^{2}$-statistic. If $I^{2}>50 \%$, the studies were considered to exhibit severe heterogeneity. If there was a significant statistical heterogeneity among the studies, the random-effects model was applied, otherwise fixed-effects model was used. The potential publication bias was assessed by using Begg's funnel plots. A $P$-value of less than 0.05 was considered statistically significant.

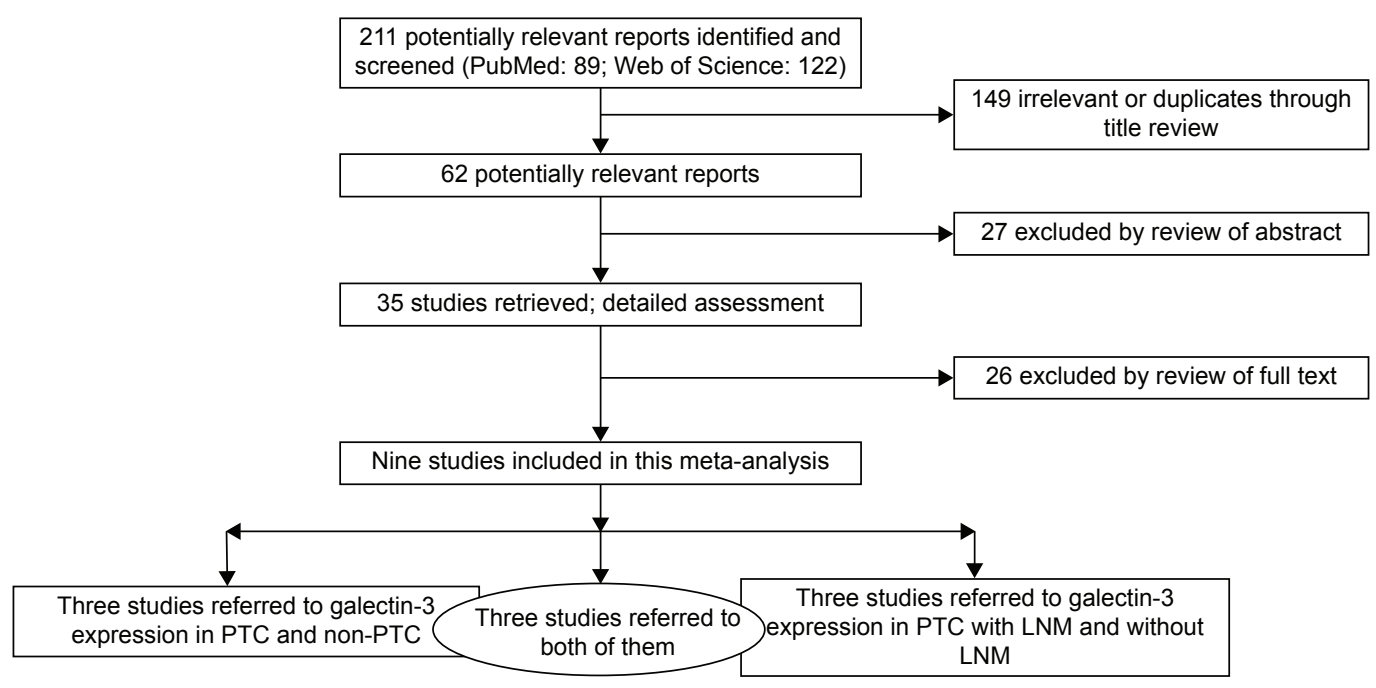

Figure I Flowchart for the selection of primary studies in this meta-analysis.

Abbreviations: PTC, papillary thyroid carcinoma; LNM, lymph node metastasis. 
Table I Characteristics of the eligible studies about galectin-3 expression in patients with or without PTC in this meta-analysis

\begin{tabular}{|c|c|c|c|c|c|c|c|c|c|}
\hline \multirow[t]{3}{*}{ First author } & \multirow[t]{3}{*}{ Year } & \multirow[t]{3}{*}{ Country } & \multirow{3}{*}{$\begin{array}{l}\text { Cancer } \\
\text { types }\end{array}$} & \multirow{3}{*}{$\begin{array}{l}\text { Total } \\
\text { number }\end{array}$} & \multirow[t]{3}{*}{ Detection method } & \multicolumn{4}{|c|}{ Galectin-3 expression } \\
\hline & & & & & & \multicolumn{2}{|c|}{ PTC } & \multicolumn{2}{|c|}{ Non-PTC } \\
\hline & & & & & & + & - & + & - \\
\hline Htwe et al ${ }^{12}$ & 2010 & Malaysia & PTC & 142 & Immunohistochemistry & 107 & I & 31 & 3 \\
\hline Selemetjev et $\mathrm{al}^{10}$ & 2015 & Serbia & PTC & 99 & Immunohistochemistry & 55 & 14 & 12 & 18 \\
\hline Gong et $\mathrm{al}^{13}$ & 2012 & People's Republic of China & PTC & 50 & Immunohistochemistry & 37 & 1 & 2 & 10 \\
\hline Lavra et a $\left.\right|^{17}$ & 2011 & Italy & PTC & 29 & Immunohistochemistry & 22 & 2 & 2 & 3 \\
\hline Barut et al ${ }^{18}$ & 2010 & Turkey & PTC & 85 & Immunohistochemistry & 52 & 3 & 10 & 20 \\
\hline Trimboli et al ${ }^{14}$ & 2015 & Italy & PTC & 19 & Immunohistochemistry & 2 & 2 & I & 14 \\
\hline
\end{tabular}

Abbreviations: +, positive expression; -, negative expression; PTC, papillary thyroid carcinoma.

\section{Results}

\section{Description of studies}

As shown in the flow diagram (Figure 1), our search terms revealed 211 articles. After reviewing the titles, 149 irrelevant articles or duplicates were excluded. After thorough inspection of the abstracts, 35 reports were excluded. Then, the full texts were read thoroughly, and a total of nine articles were finally included in the current meta-analysis. ${ }^{10-18} \mathrm{~A}$ total of 424 patients from six eligible studies that provided data about galectin-3 expression in PTC and non-PTC patients were included. A total of 378 patients from six eligible studies that provided data about galectin-3 expression in PTC patients with and without LNM were included. Studies were conducted in Malaysia, Serbia, People's Republic of China, Italy, Turkey, Korea, and Australia. Immunohistochemistry technique was used in all the studies. The main characteristics of the eligible studies are summarized in Tables 1 and 2 .

\section{Meta-analysis results of the relationship among galectin-3, PTC, and LNM}

Fixed-effects model was adopted in analyzing the expression rates of galectin-3 in PTC, since there was no significant heterogeneity among the studies $\left(I^{2}<50 \%\right)$ (Figures 2 and 3 ). Figure 2 directly reflects significant difference in the positive expression of galectin-3 between the group with PTC and the compare group with other thyroid lesions. Galectin-3 was found to be a highly sensitive $(275 / 424,64.86 \%)$ marker in the diagnosis of PTC, but was found to be expressed in only few cases involving other types of thyroid lesions (58/424, $13.68 \%$ ). The odds ratio, expressed as PTC group versus other thyroid lesions group, was 13.97 ( $95 \%$ confidence interval $[\mathrm{CI}]: 7.51-26.01, P<0.00001)$. Figure 3 shows that the positive expression rates of galectin-3 in PTC patients with LNM were higher than those in the PTC patients without LNM. The odds ratio, expressed as positive galectin-3 group in PTC patients with LNM versus negative galectin-3 group in PTC patients without LNM, was 2.55 (95\% CI: 1.09-5.97, $P=0.03$ ). This result demonstrated that PTC patients with positive expression of galectin-3 were more prone to LNM.

\section{Publication bias}

Begg's test was used to assess the publication bias. Figure 4 shows the Begg's funnel plot (pseudo 95\% CI) for the expression of galectin-3 in PTC. Figure 5 shows the Begg's funnel plot for the expression of galectin-3 in PTC patients with or without LNM. No significant publication bias was observed. This result showed that this meta-analysis was meaningful and galectin-3 was helpful for the diagnosis of PTC and prognosis for PTC patients. As a result, the conclusion of this meta-analysis had high credibility.

Table 2 Characteristics of the eligible studies about galectin-3 expression in PTC patients with or without LNM in this meta-analysis

\begin{tabular}{|c|c|c|c|c|c|c|c|c|c|}
\hline \multirow[t]{3}{*}{ Study } & \multirow[t]{3}{*}{ Year } & \multirow[t]{3}{*}{ Country } & \multirow{3}{*}{$\begin{array}{l}\text { Cancer } \\
\text { types }\end{array}$} & \multirow{3}{*}{$\begin{array}{l}\text { Total } \\
\text { number }\end{array}$} & \multirow[t]{3}{*}{ Detection method } & \multicolumn{4}{|c|}{ Galectin-3 expression } \\
\hline & & & & & & \multicolumn{2}{|c|}{$\begin{array}{l}\text { With } \\
\text { LNM }\end{array}$} & \multicolumn{2}{|c|}{$\begin{array}{l}\text { Without } \\
\text { LNM }\end{array}$} \\
\hline & & & & & & + & - & + & - \\
\hline Selemetjev et a $\left.\right|^{10}$ & 2015 & Serbia & PTC & 69 & Immunohistochemistry & 12 & 3 & 43 & II \\
\hline Gong et $\mathrm{al}^{13}$ & 2012 & People's Republic of China & PTC & 38 & Immunohistochemistry & 6 & 0 & 31 & I \\
\hline Lee and Lee ${ }^{15}$ & 2013 & Korea & PTC & 168 & Immunohistochemistry & 27 & 138 & 0 & 3 \\
\hline Salajegheh et al ${ }^{16}$ & 2014 & Australia & PTC & 58 & Immunohistochemistry & 9 & 0 & 18 & 31 \\
\hline Lavra et al ${ }^{17}$ & 2011 & Italy & PTC & 24 & Immunohistochemistry & 8 & 0 & 14 & 2 \\
\hline Liu et al" & 2014 & People's Republic of China & PTC & 21 & Immunohistochemistry & 7 & 2 & 8 & 4 \\
\hline
\end{tabular}

Abbreviations: PTC, papillary thyroid carcinoma; LNM, lymph node metastasis. 


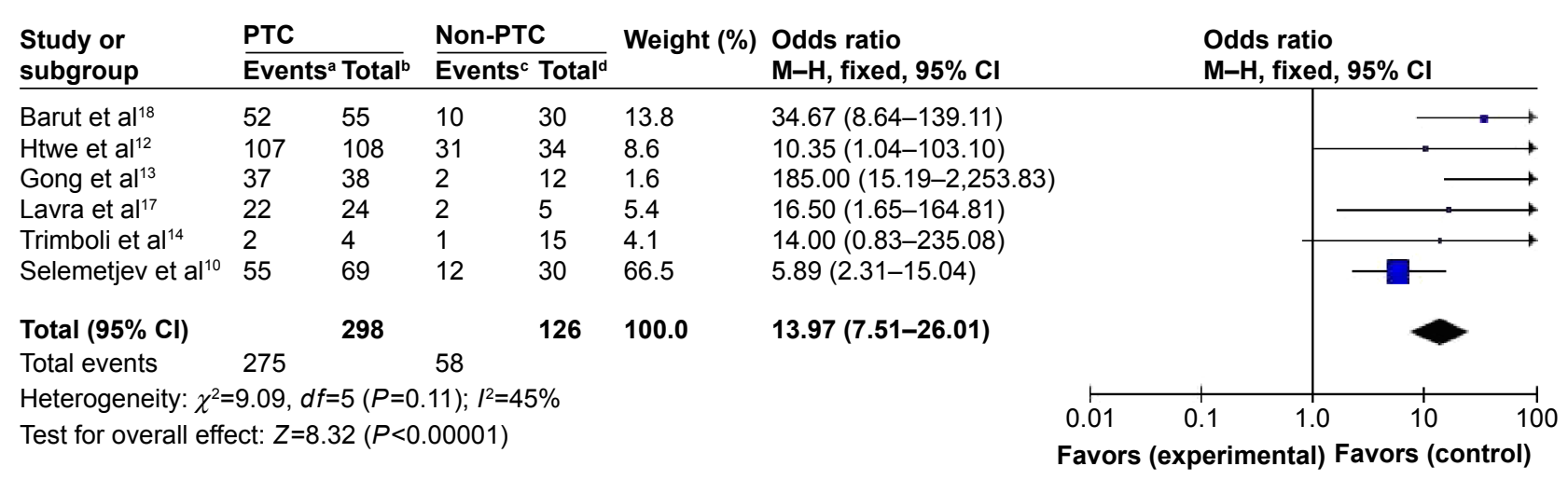

Figure 2 Forest plot for the expression of galectin-3 in patients with PTC or non-PTC.

Notes: a Number of PTC patients with positive expression of galectin-3. ${ }^{\circ}$ Total number of patients with PTC. 'Number of patients without PTC with positive expression of galectin-3. 'Total number of patients without PTC.

Abbreviations: PTC, papillary thyroid carcinoma; Cl, confidence interval; df, degrees of freedom; M-H, Mantel-Haenszel.

\section{Discussion}

Thyroid fine needle aspiration biopsy (FNAB) has been regarded as the most accurate method to distinguish between malignant and benign lesions of the thyroid gland. Research showed that after FNAB cytopathology, 5\% of the thyroid lesions are malignant and $60 \%-70 \%$ are benign, whereas in $10 \%-30 \%$ of the cases the diagnosis remains uncertain or is regarded as suspicious malignant. Of these uncertain or suspicious malignant cases, only $20 \%-25 \%$ is approved to be thyroid cancer after postoperative pathology. Therefore, $70 \%-75 \%$ of the cases may not need to undergo surgery. ${ }^{19}$ Thus, there arises a need for an immunohistochemical marker to improve the diagnostic accuracy of thyroid lesions.

Recently, galectin-3 has received significant attention for its utility as a diagnostic marker for thyroid cancer and represents the most well-studied molecular candidate for the diagnosis of thyroid cancer. This immune marker has been implicated in the regulation of normal cellular proliferation, apoptosis, malignant transformation, and the metastasis of cancer cells. Many immunohistochemical studies found that galectin-3 was differentially expressed in thyroid carcinoma compared with benign and normal thyroid specimens, suggesting that galectin-3 is a good diagnostic marker for thyroid cancer. Galectin-3 expression has been suggested as a marker having a relatively higher sensitivity and specificity in thyroid malignancies, especially in papillary carcinomas. ${ }^{6,20-22}$ In a large multicenter study by Bartolazzi et al, the sensitivity and specificity of galectin-3 in thyroid carcinomas were $99 \%$ and $98 \%$, respectively. ${ }^{23}$ In the present study, we observed a significant difference in the positive expression of galectin-3 between the group with PTC and the compare group with other thyroid lesions. Galectin-3 was found to be a highly sensitive $(275 / 424,64.86 \%)$ marker in the diagnosis of PTC, but was found to be expressed only in few cases involving other types of thyroid lesions $(58 / 424,13.68 \%)$. The odds

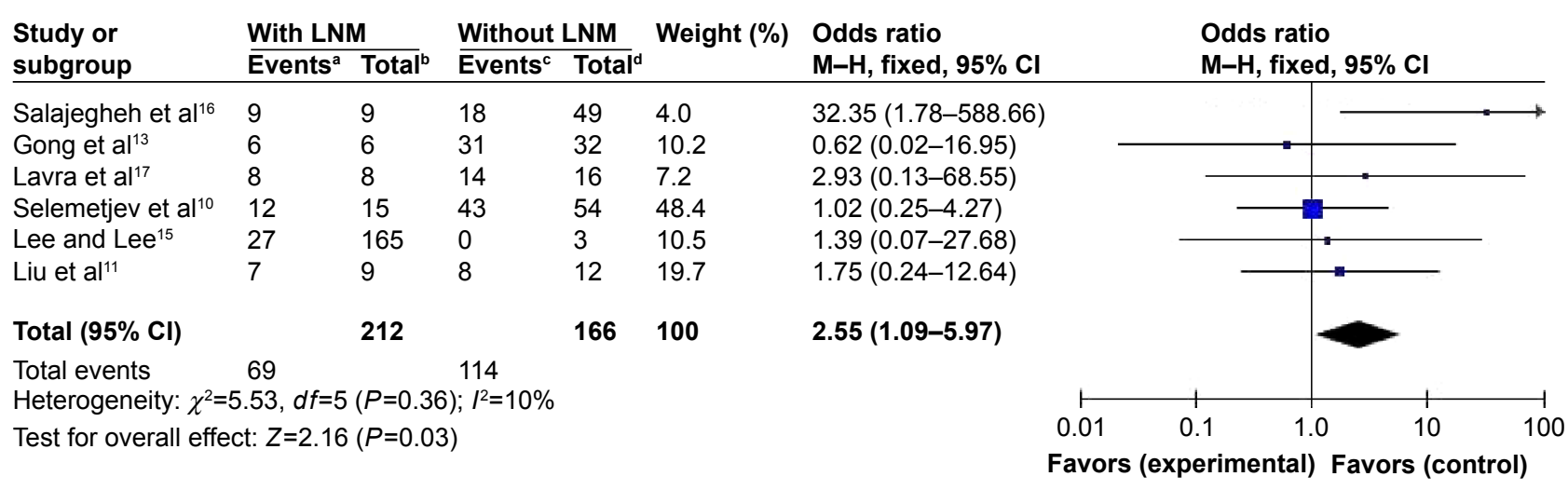

Figure 3 Forest plot for the expression of galectin-3 in PTC patients with or without LNM.

Notes: aNumber of PTC patients with LNM and positive expression of galectin-3. ${ }^{\circ}$ Total number of PTC patients with LNM. 'Number of patients without PTC and LNM with positive expression of galectin-3. ${ }^{\mathrm{d}}$ Total number of patients without PTC and LNM.

Abbreviations: PTC, papillary thyroid carcinoma; LNM, lymph node metastasis; Cl, confidence interval; df, degrees of freedom; M-H, Mantel-Haenszel. 


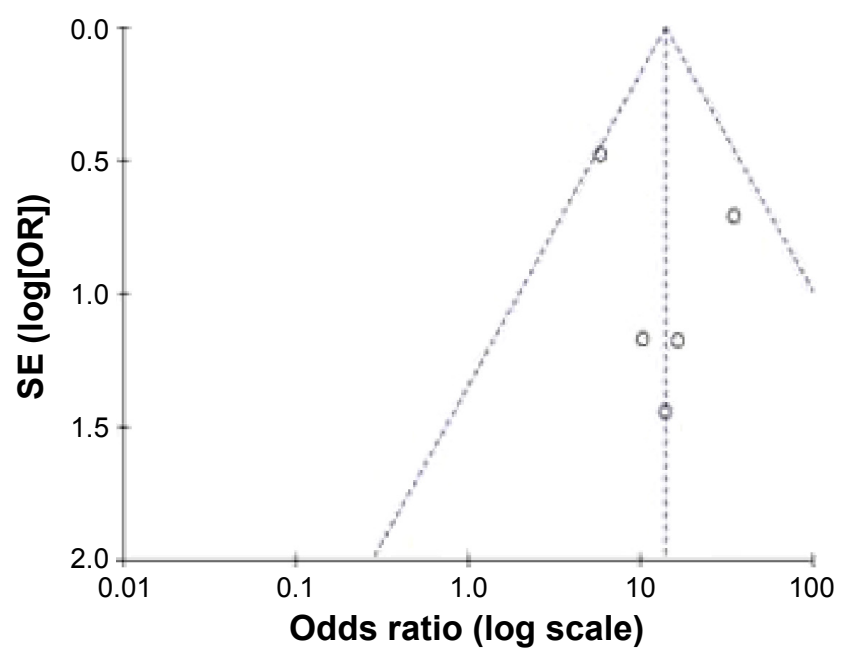

Figure 4 Begg's funnel plot for the expression of galectin-3 in patients with PTC or non-PTC.

Abbreviations: PTC, papillary thyroid carcinoma; SE, standard error of the mean; OR, odds ratio.

ratio, expressed as PTC group versus other thyroid lesions group, was 13.97 (95\% CI: 7.51-26.01, $P<0.00001$ ). Our studies also showed that the positive expression rates of galectin-3 in PTC patients with LNM were higher than those in PTC patients without LNM. The odds ratio, expressed as positive galectin-3 group in PTC patients with LNM versus negative galectin-3 group in PTC patients without LNM, was 2.55 (95\% CI: 1.09-5.97, $P=0.03$ ). These results were consistent with the previous studies. Thus, galectin-3 could become a potentially useful immunomarker to distinguish between thyroid benign and malignant lesions. PTC patients with positive expression of galectin-3 were more prone to

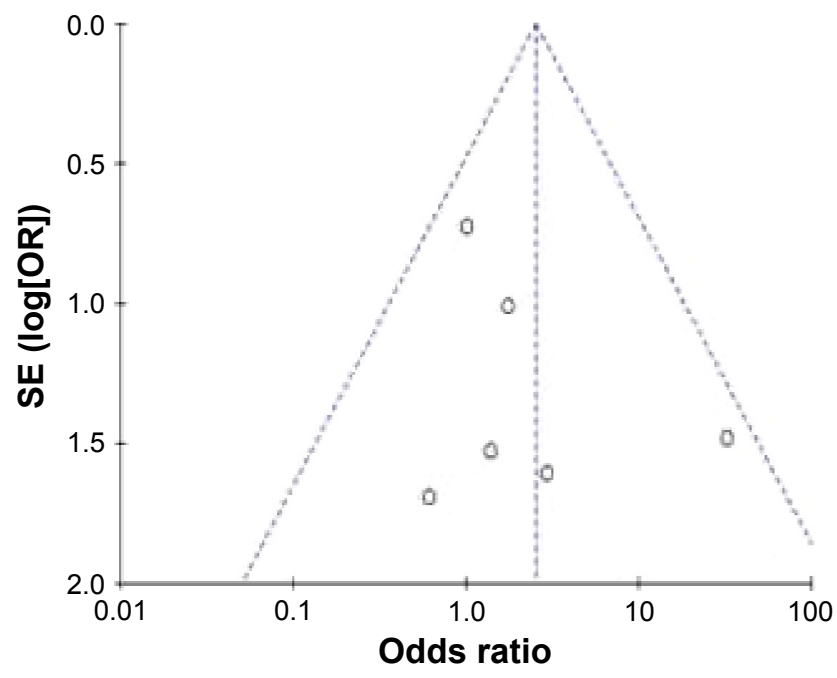

Figure 5 Begg's funnel plot for the expression of galectin-3 in PTC patients with or without LNM.

Abbreviations: PTC, papillary thyroid carcinoma; LNM, lymph node metastasis: $\mathrm{SE}$, standard error of the mean; OR, odds ratio.
LNM. As a result, galectin-3 showed more value and sensitivity in terms of diagnosis and prognosis of PTC than for other types of thyroid lesions.

\section{Conclusion}

This meta-analysis demonstrated that galectin-3, a potential immunomarker, is accurate in making a preoperative distinction between PTC and non-PTC patients. In addition, galectin-3 may provide more significant contributions in distinguishing PTC with or without LNM. We suggest a larger size and better design studies should be conducted to confirm our results.

\section{Disclosure}

The authors report no conflict of interests in this work.

\section{References}

1. Erdem H, Gündogdu C, Sipal S. Correlation of E-cadherin, VEGF, COX-2 expression to prognostic parameters in papillary thyroid carcinoma. Exp Mol Pathol. 2011;90(3):312-317.

2. Rabinovich GA. Galectin-1 as a potential cancer target. Br J Cancer. 2005;92(7):1188-1192.

3. Chiariotti L, Salvatore P, Frunzio R, et al. Galectin genes: regulation of expression. Glycoconj J. 2004;19(7-9):441-449.

4. Dumic J, Dabelic S, Flögel M. Galectin-3: an open-ended story. Biochim Biophys Acta. 2006;1760(4):616-635.

5. Cvejic D, Savin S, Petrovic I, et al. Galectin-3 expression in papillary microcarcinoma of the thyroid. Histopathology. 2005;47(2):209-214.

6. Kawachi K, Matsushita Y, Yonezawa S, et al. Galectin-3 expression in various thyroid neoplasms and its possible role in metastasis formation. Hum Pathol. 2000;31(4):428-433.

7. Lin TW, Chang HT, Chen $\mathrm{CH}$, et al. Galectin-3 binding protein and galectin-1 interaction in breast cancer cell aggregation and metastasis. J Am Chem Soc. 2015;137(30):9685-9693.

8. Punt S, Thijssen VL, Vrolijk J, et al. Galectin-1, -3 and -9 expression and clinical significance in squamous cervical cancer. PLoS One. 2015; 10(6):e0129119.

9. Xin M, Dong XW, Guo XL. Role of the interaction between galectin-3 and cell adhesion molecules in cancer metastasis. Biomed Pharmacother. 2015;69C:179-185.

10. Selemetjev SA, Savin SB, Paunovic IR, et al. Changes in the expression pattern of apoptotic molecules (galectin-3, Bcl-2, Bax, survivin) during progression of thyroid malignancy and their clinical significance. Wien Klin Wochenschr. 2015;127(9-10):337-344.

11. Liu Z, Li X, Shi L, Maimaiti Y, et al. Cytokeratin 19, thyroperoxidase, HBME-1 and galectin-3 in evaluation of aggressive behavior of papillary thyroid carcinoma. Int J Clin Exp Med. 2014;7(8): 2304-2308.

12. Htwe TT, Karim N, Wong J, et al. Differential expression of galectin-3 in advancing thyroid cancer cells: a clue toward understanding tumour progression and metastasis. Singapore Med J. 2010;51(11): 856-859.

13. Gong L, Chen P, Liu X, et al. Expressions of D2-40, CK19, galectin-3, VEGF and EGFR in papillary thyroid carcinoma. Gland Surg. 2012; 1(1):25-32.

14. Trimboli P, Guidobaldi L, Amendola S, et al. Galectin-3 and HBME-1 improve the accuracy of core biopsy in indeterminate thyroid nodules. Endocrine. Epub 2015 Jul 4.

15. Lee YM, Lee JB. Prognostic value of epidermal growth factor receptor, p53 and galectin-3 expression in papillary thyroid carcinoma. J Int Med Res. 2013;41(3):825-834. 
16. Salajegheh A, Dolan-Evans E, Sullivan E, et al. The expression profiles of the galectin gene family in primary and metastatic papillary thyroid carcinoma with particular emphasis on galectin-1 and galectin-3 expression. Exp Mol Pathol. 2014;96(2):212-218.

17. Lavra L, Rinaldo C, Ulivieri A, et al. The loss of the p53 activator HIPK2 is responsible for galectin-3 overexpression in well differentiated thyroid carcinomas. PloS One. 2011;6(6):e20665.

18. Barut F, Onak Kandemir N, Bektas S, et al. Universal markers of thyroid malignancies: galectin-3, HBME-1, and cytokeratin-19. Endocr Pathol. 2010;21(2):80-89.

19. Gómez Sáez JM. Diagnostic and prognostic markers in differentiated thyroid cancer. Curr Genomics. 2011;12(8):597-608.

20. Bernet VJ, Anderson J, Vaishnav Y, et al. Determination of galectin-3 messenger ribonucleic acid overexpression in papillary thyroid cancer by quantitative reverse transcription-polymerase chain reaction. J Clin Endocrinol Metabol. 2002;87(10):4792-4796.
21. Volante M, Bozzalla-Cassione F, Orlandi F, Papotti M. Diagnostic role of galectin-3 in follicular thyroid tumors. Virchows Arch. 2004; 444(4):309-312.

22. Than TH, Swethadri GK, Wong J, et al. Expression of galectin-3 and galectin-7 in thyroid malignancy as potential diagnostic indicators. Singapore Med J. 2008;49(4):333-338.

23. Bartolazzi A, Gasbarri A, Papotti M, et al. Application of an immunodiagnostic method for improving preoperative diagnosis of nodular thyroid lesions. Lancet. 2001;357(9269):1644-1650.
OncoTargets and Therapy

\section{Publish your work in this journal}

OncoTargets and Therapy is an international, peer-reviewed, open access journal focusing on the pathological basis of all cancers, potential targets for therapy and treatment protocols employed to improve the management of cancer patients. The journal also focuses on the impact of management programs and new therapeutic agents and protocols on

\section{Dovepress}

patient perspectives such as quality of life, adherence and satisfaction. The manuscript management system is completely online and includes a very quick and fair peer-review system, which is all easy to use. Visit http://www.dovepress.com/testimonials.php to read real quotes from published authors. 